\title{
SPORT AS PART OF THERAPY AND REHABILITATION OF PARAPLEGICS
}

\author{
By Professor Marian Weiss, M.D. and J. Beck, Ph.D. \\ Rehabilitation Department, Warsaw School of Medicine, Konstancin
}

\section{INTRODUCTION}

LONG-STANDING observations of the good effects of various sport exercises in the process of social and vocational rehabilitation of paraplegics, have induced us to consider all the aspects of this problem in the medical rehabilitation programme. Guttmann and Michaelis (1970) demonstrated that regular practice of sports and special training in various branches of performance sports, for competition within the framework of the Stoke Mandeville Olympic Games-helps reduce the number of complications and varieties of disturbances observed in the pathology of man with damaged spinal cord. There is no doubt whatever that regular exercises are a factor essential for helping compensatory functions during the treatment process.

Our intention is to demonstrate in this paper the value of sport in the programme of medical rehabilitation. We are specially interested in the influence various exercises have in the preparation for continuation of gymnastics and remedial exercises in a determined branch of sport.

\section{NEUROLOGICAL BASIS FOR EARLY APPLICATION OF SPORT IN THE REHABILITATION OF PARAPLEGICS}

It is generally known that spinal cord injuries lead to trophic changes and other peripheral disturbances. The slowed down blood circulation in the paralysed extremities, changes in basic metabolism, alterations in the skeletal system and other tissues-require exercises to help stop progression of pathological changes and lead to reconstruction of functions.

Spinal cord injuries following vertebral trauma, and the grave condition of the patient after accident, make intensive exercises impossible. Within that time - over Io days-only passive exercises are applied as well as electrostrimulation and other procedures enabling restoration of functions and preventing occurrence of peripheral venostasis.

After this initial period, called the acute stage, there follows a process of natural repair within the spinal cord; the formation of scars, absorption of haematomas and necrotic tissues, etc.

It is not enough, however, to carry out passive exercises and apply electrostimulation. It is indispensable to start application of other forms of biological stimulation in the regeneration process of the spinal cord itself.

From the pathophysiological aspect, any procedure should always aim at:

I. Ensuring the complete range-of course within the possibilities existingof proprio- and interoceptive stimuli from the periphery.

2. Stepping up blood supply to the paralysed extremities, obtained only by means of increased general effort. 
PAPERS READ AT THE ANNUAL SCIENTIFIC MEETING, I 972

3. Providing correct gas exchange, especially by the intermediary of the pulmonary system.

4. Ensuring normal gravity stimuli; this is of great importance in preventing regressive changes in the bone and joint system.

5. Enabling the patient to reach psychological adjustment that would permit him to counteract the cerebral shock period caused by the sudden cutting off of the activating stimuli.

\section{METHOD ADOPTED}

Studies on early application of exercises were conducted on the clinical material of patients admitted to the Konstancin Rehabilitation Institute's acute spinal cord injuries department. These exercises included:

(a) Training preparatory for various sport branches

(b) training enabling the choice of an appropriate type of sport, one that would be of interest for the patient also after dehospitalisation

(c) choice of such a sport that could be practised in the patient's individual social conditions

Our assumption was that these exercises could replace the less interesting physiotherapeutic measures such as, progressive resistance exercises, exercises with medicine balls, springs, etc.

A patient with spinal cord damage in the cervical or upper thoracic section must be prepared to operate a wheelchair by himself. This is possible at an adequate compensatory efficiency of certain other parts of the body, especially if the remaining dynamic complexes are strong enough, e.g. the shoulder, or elbow joint.

There can be no independent operation of the wheelchair without training, for this purpose, of the wrist and palm muscles. This is why there must be gradual appropriate increase applied in resistance with many isometric elements. Simultaneous expansion and well-elaborated dosage of treatment are necessary to reach improvement within the shortest time. Overcompensation of those muscle groups which the patient did not have to use previously should be tried to be obtained.

Considering the condition of paraplegics, all their functions must base on the upper dynamic complexes of the body, and the training must take this into account.

In the case of paraplegics with lower spinal cord damage, from D.I I, D.I2 to L.3, exercises are expanded to include the trunk muscles, especially all the abdominal muscle groups.

It is clear that early physiotherapy, dynamising the shoulder and trunk, requires radical local approach to the broken spine.

The method we have introduced in our Institute, is stabilisation by means of bone transplant of the cervical spine, sometimes with total resection of the fractured vertebral body. We use transplants from the fibula or ilium. In case of fracture in the lower spine sections (thoraco-lumbar) there is stabilisation of the spine by means of two rows of springs under high tension. With this technique we can surgically follow Guttmann's ideas. Our pilot group of Ioo cases handled by this technique should permit gradual correction of the broken spine, with simultaneous good stabilisation thereof. 
Full training begins after Io days of hospitalisation in our Institute. If the general condition of the patient is good, exercises are conducted also during the acute stage, as it is possible to raise the patient to a vertical position on a special bed as early as 4 days after operation.

A set of special exercises is worked out at that time, permitting the triceps of the arm on which the patient will have to support himself later on, when using elbow crutches to be strengthened.

Preparatory training to sports begins with a psychological analysis as to the type of sport the patient would prefer. Weight lifting and athletics are suggested in the first place, as swimming is possible only in certain towns and under special conditions. There is no adequate network of swimming pools in Poland yet, without architectural barriers to paraplegics.

After deciding on the type of sport, the instructor begins preparatory exercises. $\mathrm{He}$ acquaints the patient with the equipment, explains the bio-mechanical value of movements at the various planes of that equipment (figs. I-3). Next comes preparatory training with the same equipment as used by normal people. Patients are shown films on sports; they are encouraged to carry out sport exercises later on, to keep up the physical condition they acquired at the Institute of Rehabilitation. They are also advised to arrange sport facilities at home and organise appropriate groups-depending on the place of residence.

This system allowed sport sections for paraplegics to be organised in practically all the larger provincial towns. Our former patients, employed later by invalids' cooperatives, created their own sport sections for paraplegics within the framework of the Polish Sport Association for the Disabled.

\section{TRAINING METHOD}

The first principle is that most of the exercises are conducted in standing position, as we are concerned with the importance of early application of the gravity stimulus. There is available for this purpose, special tables and beds, both for rotation of the patient, and for gradual tilting. The instructor gives the patient certain items of equipment: a bow, grenade or javelin, medicine ball, or other items for training in handling weights, a ball for throwing, badminton or table tennis bats. The patient is placed on an appropriate table in the gymnasium, at small distance from the target and is encouraged to start training.

Early on in the training the patient is secured by several straps; this external stabilisation is gradually reduced, enabling new muscle groups to be exercised. A 45-minute training session is conducted at least twice a day. Moreover, when the patient is in bed and is fitted with a complete system of blocks, weights, springs and medicine balls, the patient exercises under the supervision of a nurse or the instructor in charge of his ward.

These exercises are conducted from the Ioth day after the accident for 6 weeks. After 6 weeks the first efficiency tests are made. The patient is acquainted with the tests in all detail (figs. 4 and 5) he is also told about the records gained at domestic competitions and at the International Olympic Games at Stoke Mandeville.

Throughout the exercises, the instructor makes all the standard dynamometric and ergometric tests. An energometric test made every 2 weeks complements the preparation of the patient to withstand effort.

Electromyographic examinations help to determine the best positions for 


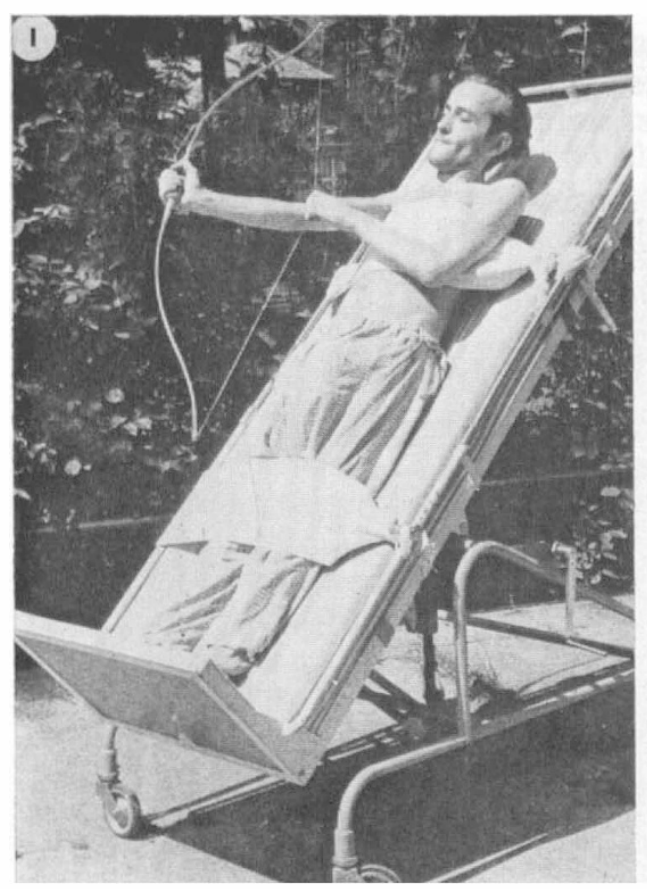

FIG. I

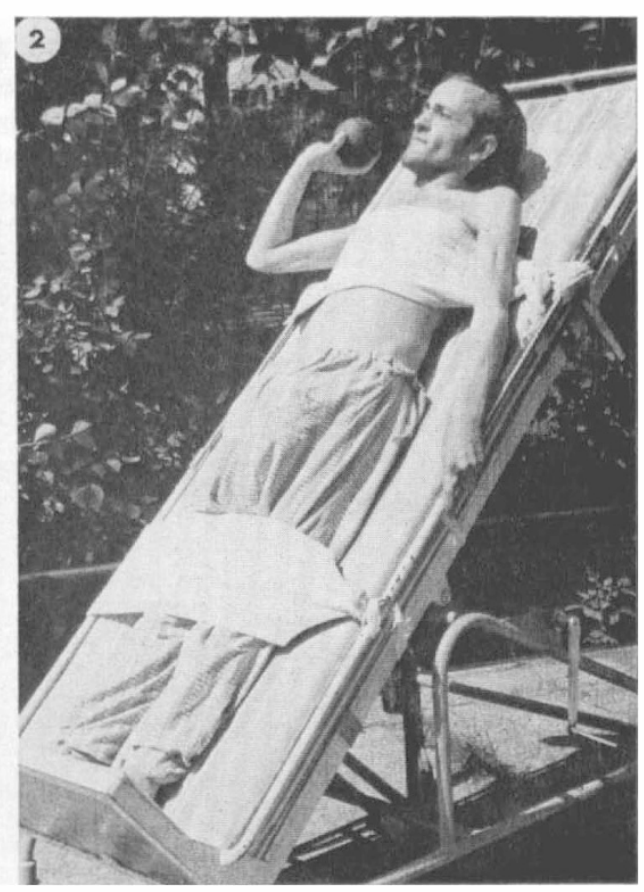

FIG. 2

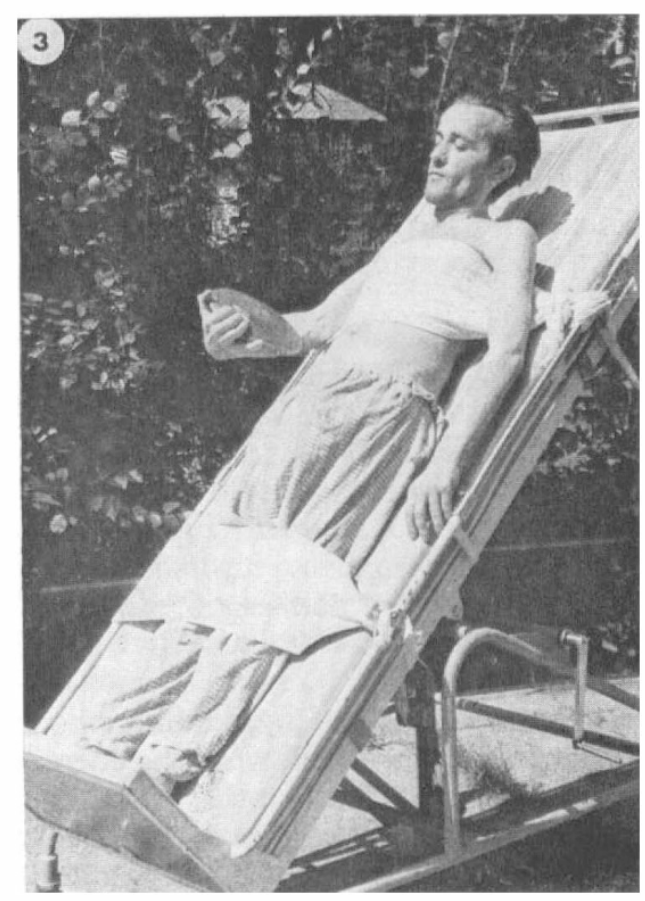

FIG. 3 
activation of the shoulder muscles and arm triceps. The intensity of exercises depends on the patient's reactions; these reactions are controlled by a team composed of: physiotherapist, psychologist and physician.

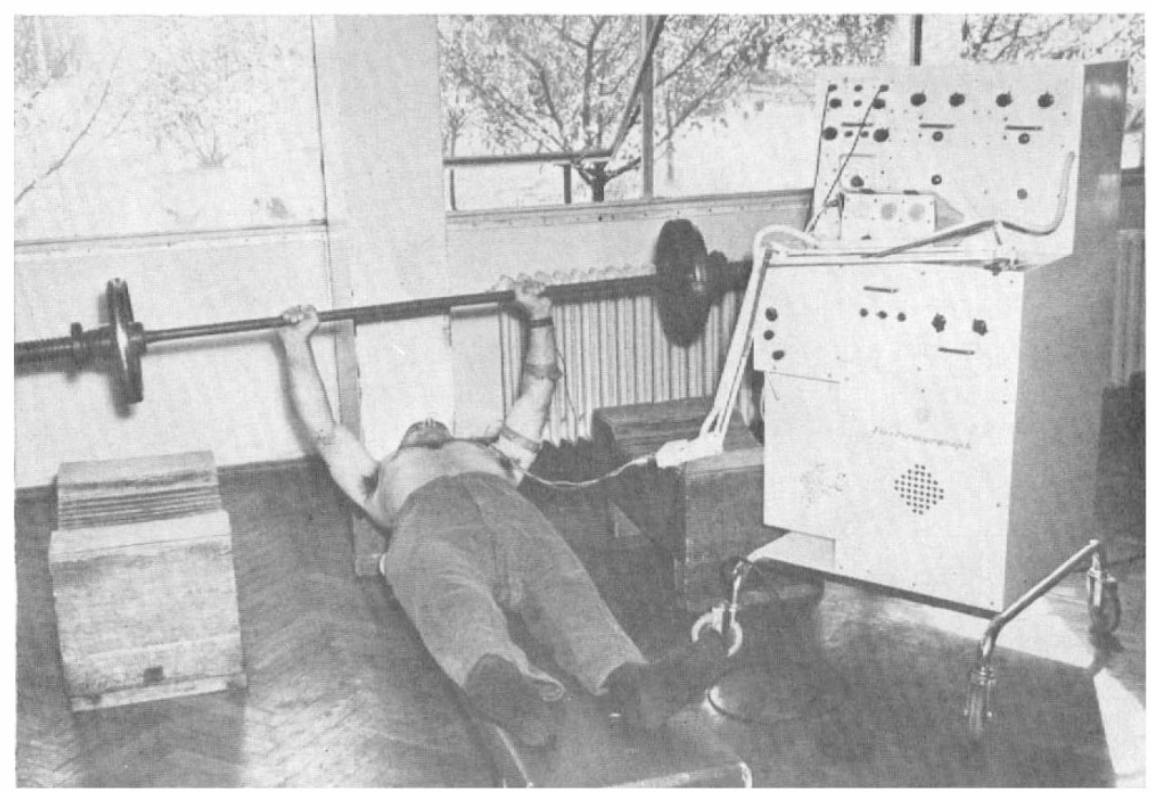

FIG. 4

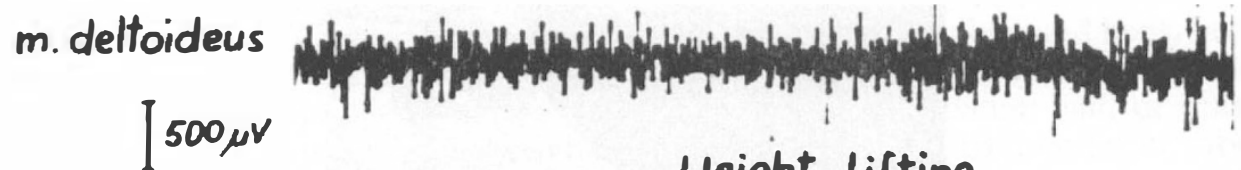

\section{Weight Lifting}

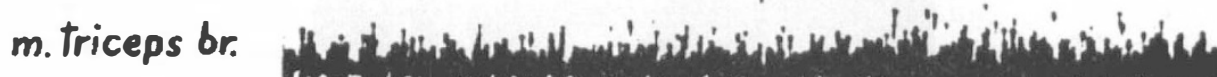

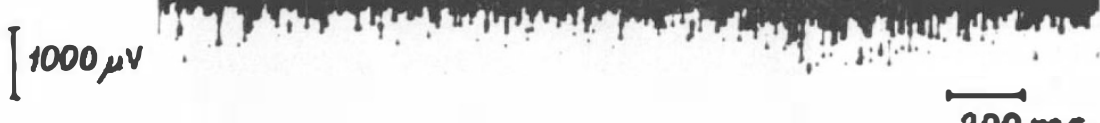

$200 \mathrm{~ms}$

The patient is first included in group C, providing 2 hours of exercises a day, after about 2-3 weeks he goes over to group B, where he attends 4 hours of exercises a day. He remains in that group for 2 months. The next stage is group A, providing a 6-hour daily programme, performed outside, or in gymnasiums or sports halls. At that time every endeavour is made to make every patient join a group of social occupations and games played in wheelchairs, Basketball in 
particular is preferred, as this trains reflex actions and permits the patient to master equilibrium in the wheelchair under various conditions.

The routine sport exercises in our centre were based on the experience of the Olympic Games training centre and also on that of the Academy of Physical Culture, Warsaw, a department of which has existed in our Institute since I95I. Instructors in various branches are at the same time methodic consultants for the sport branches concerned.

\section{THE RESULTS ACHIEVED}

Clinical examinations revealed that by first applying preparatory sport training and next normal exercises in various sports a roo per cent. faster increase in the strength of upper extremities and shoulder muscles was achieved than with conventional bedside treatment. Especially marked was the improvement in the trophic condition of the lower extremities and of the entire body. Our intention was, as a rule, to utilise fatigue effects for increasing the blood flow through the heart and also for stepping up respiration. We tried to reach 30 breaths a minute, which we accepted as being the respiratory cycle enabling full ventilation and acceleration of metabolic processes, also in the periphery.

No spasticity in flexion was found in the patients observed. And, if there actually was spasticity, it was of the extensor type. This was presumed to be due to the early applied verticalisation and the re-organisation of the spinal cord under the influence of gravity stimuli, of great significance for ensuring proprioception, and the flow of stimuli to the injured site.

In a few patients admitted as being completely paralysed, partial restoration of functions was observed. In one case, even complete restoration. We were unable to explain this mechanism; maybe the reason was that, in these patients, the neurological examination had not been adequate as, in acute cases, such examinations are not always objective enough.

In acute spinal cord injuries we cannot make either spinal cord arteriography or myelography, as such additional loading is not permissible. According to rules laid down by Guttmann, we avoid adding surgery shock to that of the spinal cord trauma.

The operation itself, consisting of setting up two rows of springs, is a simple procedure of short duration, and not injurious to the spine in any way. Laminectomy is not performed, as a rule. The muscles, even if partially damaged, are replaced by elastic springs, ensuring good stabilisation of the broken spine and enabling its subsequent slow, but steady correction.

The important effect of this therapeutic programme is the almost complete elimination of the acute psychologic reactions observed in the paraplegics treated for many months in a recumbent position.

Our patients accepted their disability easily, the more so as they started ambulation training - as soon as 4 weeks after. This is, of course, applicable only to patients in whom the spinal cord damage was below D.8. All those with injuries at a higher level operated wheelchairs with ease and their daily routine was complemented by sport exercises.

We now feel sure that early introduction of sport training and devising the entire physiotherapy programme for strengthening muscles by means of such exercises produce very good results. Patients have stronger motivation to train 
in such instances. They take up a variety of occupations on their own, as they are interested in having better results in efficiency tests. They understand these exercises to be necessary. They are, moreover, encouraged by the fact that practice of sports will bring them nearer to sport life at home. Our patients often train with such obstinacy that we have to interfere.

The conventional teaching of our physiotherapists is a great obstacle in introducing this system. This is why we employ in our Institute sport instructors who can activate patients better as they have been specially trained to do so.

The traditions of Polish light athletics and the development of the Olympic Training Centre have also allowed us to step up our respective activities. We consider the Olympic Training Centre's programme and Stoke Mandeville Olympic Games which, although arranged for handicapped people, have begun to play an increasingly greater role in the process of social rehabilitation of invalids with spinal cord injuries.

To reach better results in sport, viewed from the social aspect, it is necessary to start training and to develop physiotherapeutic methods on the basis of experiences of Higher Schools of Physical Culture, and of Physical Training Centres for Instructors.

\section{CONCLUSIONS}

I. Preparation for practising some type of sport should begin at the time of the natural repair within Io days after the accident.

2. This is possible only by good stabilisation of the damaged spine section.

3. Early training in sports permits loading of the body to the level indispensable for reaching the desired therapeutic effect. This may be a loading around the physiological 'second wind' limit.

4. Early starting of exercises within determined sport branches, properly chosen for para- and tetraplegics, encourages invalids to practise sports. This is, of course, of fundamental importance for stabilising the rehabilitation effects.

5. Early introduction of sport disciplines into treatment of paraplegia eliminates the psychological troubles so typical in our practice of rehabilitation of paraplegics.

\section{REFERENCES}

Guttmann, L. \& Michaelis, L. S. (1970). Proceedings of the Annual Scientific Meeting of the International Medical Society of Paraplegia, 28 to 30 July 1969, held at Stoke Mandeville Hospital, Aylesbury. Paraplegia, 8, 67-9.

Jougers, J. J., Tricot, A. \& JoYe, H. (I970). Cout métaboloque et effets physiologiques de l'effort et du sport chez les handicapés. Schwiez $Z$ Sportmed. 18, 3, I I7-I25.

NiCKERSON, E. (I97I). Some correlates of adjustment by paraplegics. Percept Motor Skills, 32, I, I I-23.

SlaŃCEv, P. \& IlIEv, I. (I97I). XVIII Svetoven Kongres po sportna medicina. Vapr Fiz Kult. 2, 124-2I7. 